\title{
Origin of Giant Radio Pulses
}

\author{
Ya. N. Istomin \\ P. N. Lebedev Physical Institute, Leninsky Prospect 53, 119991 Moscow, \\ Russia
}

\begin{abstract}
A model for the origin of giant radio pulses is suggested. Radio emission is generated by the electric discharge taking place due to the magnetic reconnection of field lines connecting the opposite magnetic poles. The reconnection occurs in the region of the light cylinder near the zero line of the magnetic field. The coherent mechanism of radiation is pure maser amplification of Alfvén waves. The radiated frequencies are found.
\end{abstract}

\section{Introduction}

The properties of giant pulse radio emission are very different from that of usual radio pulsar radiation. They are (Hankins et al. 2003):

1. High intensity. The brightness temperature for this emission can be as high as $10^{37} \mathrm{~K}$. Such a value approaches the temperature for the human-made generators of radio emission.

2. Short duration. There are pulses observed to last for $2 \times 10^{-9} \mathrm{sec}$.

3. Narrow spectrum. The width of the spectrum is of the order of the frequency band, $\Delta \nu \simeq \nu$.

4. Polarization. The pulses have a strong circularly polarized component.

5. $\log N-\log S$ dependence. For giant pulses, the distribution of the number of pulses $N$ having intensity greater than $S$ has the power law shape $N(>S) \propto S^{-\alpha}$. Notice that for the usual radio emission this distribution is exponential.

All these differences of giant pulses compared to the usual radio pulses indicate a different emission mechanism than that for periodic pulsar radio emission.

Here we will discuss the giant radiation from two bright sources: the Crab pulsar B0531+21 and the millisecond pulsar B1937+21. The intensity of giant radio emission from these pulsars is $2-3$ orders of magnitude greater than the intensity of usual radiation from those pulsars. They have quite different spin parameters, period $P$ and deceleration $\dot{P}$. Consequently, they have different values of magnitudes of magnetic field at the stellar surface, $B_{0}$. For the Crab pulsar, $B_{0} \simeq 4 \times 10^{12}$ gauss, but for PSR B1937+21, $B_{0} \simeq 4 \times 10^{8}$ gauss. However, if we estimate the values of the magnetic field at the light cylinder, $B_{L}=B_{0}(2 \pi R / P c)^{3}$, then they turn out to be similar, $B_{L} \simeq 10^{6}$ gauss. That is the highest value of this parameter among the radio pulsars. Another property common to these pulsars is that they both have interpulses. This indicates that the inclination angles $\chi$, i.e., angles between the axis of pulsar rotation and 
the axis of magnetic dipole, are of the order of $90^{\circ}, \chi \simeq \pi / 2$. In this case an observer sees both magnetic poles of the neutron star. The angle $\chi$ determines the structure of the pulsar magnetosphere. The plasma charge density, called Goldreich-Julian density (1969), $\varrho_{G J}=-(\mathbf{B} . \boldsymbol{\Omega}) / 2 \pi c$, is proportional to the angle between the vectors of magnetic field $\mathbf{B}$ and the axis of rotation $\boldsymbol{\Omega}, \boldsymbol{\Omega}=$ $2 \pi / P$. The magnetosphere is divided into four sectors, where $\varrho_{G J}$ has opposite signs. In the case $|\chi-\pi / 2|<(\Omega R / c)^{1 / 2}$ the surface where $\varrho_{G J}=0$ is inside the polar caps of size $R_{0} \simeq R(\Omega R / c)^{1 / 2}$ ( $R$ is the neutron star radius). The magnetic field line, starting from one polar cap with definite value of $\varrho_{G J}$, ends in second cap with the opposite value of $\varrho_{G J}$. This means that the polar magnetic field line has the opposite values of electric potential $\Psi$ at its ends near the stellar surface.

\section{Reconnection}

Let us now calculate the value of electric potential of the polar magnetosphere, $\Psi$, in the frame rotating together with the neutron star for the case $\chi=\pi / 2$. It is determined by the solution to the Laplace equation

$$
\triangle \Psi=-4 \pi\left(\varrho-\varrho_{G J}\right)
$$

with the boundary condition $\Psi=0$ on the star surface at $0<r<R_{0}$ and on the boundary of polar region $r=R_{0}, z>0$. Here $r, z, \phi$ are the cylindrical coordinates. The solution of the Laplace equation gives

$$
\Psi=\sum_{n=1}^{\infty} \frac{6(1-i) \Omega B_{0} R_{0}^{3}}{c R \mu_{n}^{3} J_{2}\left(\mu_{n}\right)}\left(1-e^{-z \mu_{n} / R_{0}}\right) J_{1}\left(\mu_{n} \frac{r}{R_{0}}\right) \cos \phi,
$$

where $\mu_{n}$ are the roots of the Bessel function of the first order $J_{1}\left(\mu_{n}\right)=0$. The maximum value of polar magnetosphere voltage $\Psi_{m}$ is achieved at the high $z \simeq$ $0.25 R_{0}$ above the star surface and near half of the polar cap radius, $r \simeq 0.5 R_{0}$,

$$
\Psi_{m}=0.42(1-i) B_{0} R\left(\frac{\Omega R}{c}\right)^{5 / 2} .
$$

The quantity $i$ is the dimensionless electric current flowing in the polar magnetosphere, $i=j / c \varrho_{G J}, i<1$. The potential $\Psi$ changes its sign on opposite sides of the polar cap. Thus, the difference of the electric potential between two ends of a magnetic field line, connecting magnetic poles, is equal to $2 \Psi_{m}$. And magnetospheric electrons and positrons, passing from one pole to another along the magnetic field line, get the high energy $\gamma m c^{2} ; \gamma \simeq 6 \times 10^{9}$ for the Crab pulsar and $\gamma \simeq 1.5 \times 10^{9}$ for PSR B1937+21.

However, the magnetic field lines, starting from the poles, do not connect with each other (see, for example, Beskin, Gurevich \& Istomin 1993). They pass above the light surface near the zero line of magnetic field. The region near the zero line is known to be unstable with respect to magnetic field reconnection. The perturbation of plasma motion near the light cylinder results in the connection of magnetic field lines starting from opposite magnetic poles. And the polar caps turn out to be linked by magnetic field lines. The reconnection process will accompany the electric discharge between the two polar caps, and plasma particle acceleration. 


\section{Maser Amplification of Plasma Waves}

During the electric discharge, plasma particles, electrons and positrons, begin to move in opposite directions along magnetic field lines inside the magnetic tube where the reconnection occurs. Such distribution of plasma particles over their momenta is unstable with respect to plasma wave excitation. This instability, the so-called two-stream instability, possesses high growth rate, $\Gamma \simeq \omega$, in the case when two streams have equaled concentrations. The characteristic plasma wave frequency $\omega$ is $\omega_{p} \gamma_{\min }^{-3 / 2} / 2$. The value of $\gamma_{\min }$ is the minimal gamma factor of plasma particle distributions generated in the process of cascade multiplication, $\gamma_{\min } \simeq 10^{2}$. The density of the plasma, born during the electric discharge, is $n_{p}=\left(\varrho_{G J} / e\right)\left(\gamma / \gamma_{\min }\right)$. The privilege for the amplification has Alfvén waves. They propagate strongly along the magnetic field lines and do not leak from the discharge tube. Moreover, they are trapped inside the tube, reflecting from the conducting surface of a neutron star. The coefficient of reflection of electromagnetic wave of frequency $\nu=\omega / 2 \pi$, from the conductor of conductivity $\sigma$, is $1-2(\nu / \sigma)^{1 / 2}$ (Landau \& Lifshitz 1960). It is very close to 1 for neutron stars, for which the conductivity is of the order of $\sigma \simeq 10^{20} \mathrm{sec}^{-1}$. For the observed radio frequencies $\nu \simeq 10^{10} \mathrm{sec}^{-1}$, the reflection coefficient is about $1-10^{-5}$. Thus, we have a maser with perfectly reflecting mirrors, working in the pulsar magnetosphere. The energy density of radio waves can achieve the energy density of the plasma near the light cylinder, $n_{p} \gamma_{m i n} m c^{2} \simeq B_{L}^{2} / 8 \pi \simeq 10^{10} \mathrm{ergs} \mathrm{cm}^{-3}$. The frequency of maser radiation is

$$
\nu=0.65 \nu_{c l} \gamma_{\min }^{-2}\left(\frac{\Omega R}{c}\right)^{1 / 4}
$$

and for Crab and PSR B1937+21 it is about $10^{9} \mathrm{~Hz}$. For a usual radio interpulse pulsar $\left(P \simeq 0.3 \mathrm{sec}, \dot{P} \simeq 10^{-15}\right)$ the characteristic giant pulse frequency is small, $\nu \simeq 10^{6} \mathrm{~Hz}$. We see that the frequency of radiation is close to the cyclotron frequency of electrons in the region of the light cylinder, $\nu \simeq \nu_{c l} / \gamma=e B_{L} / m c \gamma$. Due to that, the polarization of generated waves has a large circular component.

\section{The $\log N-\log S$ Distribution}

As we mentioned in the introduction, the dependence of the number of pulses $N$ having intensity larger than $S$ has a power law shape, $N \propto S^{-\alpha}$, for giant pulses. The power law indices $\alpha$ are different for the Crab and PSR B1937+21, $\alpha=2.3$ for Crab, and $\alpha=1.5$ for B1937+21. Let us calculate the value of $\alpha$. The radio intensity $S$ is proportional to the power of plasma generated inside the discharge tube, $S \propto c s \Psi_{m} \varrho_{G J}$. The quantity $s$ is the area of the magnetic tube where the reconnection takes place. The values of $\Psi$ and $\varrho_{G J}$ are the constant characteristics of a given pulsar, i.e. $S \propto s$. On the other hand, the energy put into the reconnection is the product of the pumping power $W$ and the time of accumulation $\tau$. It is equal to the plasma energy density $\varepsilon$ in the region of the light cylinder multiplied by the reconnection volume $V \simeq s^{3 / 2}$. If $\varepsilon=$ constant, then $\tau \propto s^{3 / 2} \propto S^{3 / 2}$. The number of observed pulses is the ratio of time of observation $T$ to the accumulation time $\tau, N \propto \tau^{-1}$. Thus, we 
obtain the power-law dependence $N \propto S^{-3 / 2}$, which corresponds to that in the pulsar PSR B1937+21. But if the value of plasma energy density in the region of magnetic reconnection $\varepsilon$ is not constant and depends on the volume $V$, then the index $\alpha$ can differ from 3/2. For example, if $\varepsilon \simeq B^{2} / 8 \pi$, and near a zero line of magnetic field, $B \propto V^{1 / 3}$, then we get the dependence $\tau \propto s^{5 / 2}$. Finally, $N \propto S^{-5 / 2}$. The obtained value of $\alpha=2.5$ is close to that for the Crab pulsar.

We see that the model proposed for generation of giant pulses explains well the observed properties of the radiation.

Acknowledgments. This work was done under support of the Russian Foundation for Fundamental Research (02-02-16762).

\section{References}

Beskin, V. S., Gurevich, A. V., \& Istomin, Ya. N. 1993, Physics of the Pulsar Magnetosphere, (Cambridge: CUP)

Goldreich, P., \& Julian, W. H. 1969, ApJ, 157, 869

Hankins, T. H., Kern, J. S., Weatherall, J. C., \& Eilek, J. A. 2003, Nature, 422, 141

Landau, L. D., \& Lifshitz, E. M. 1960, Electrodynamics of Continuous Media, (Oxford: Pergamon) 